\title{
Almost sure functional central limit theorems for multiparameter stochastic processes
}

\author{
E.B.Czerebak-Morozowicz ${ }^{1}$, Z.Rychlik ${ }^{2}$, M.Urbanek $^{2}$ \\ 1 Department of Mathematics, Technical University of Rzeszów \\ ul. Wincentego Pola 2, 35-959 Rzeszów, Poland \\ 2 Institute of Mathematics, Maria Curie-Skłodowska University \\ pl. Marii Curie-Skłodowskiej 1, 20-031 Lublin, Poland
}

Received January 31, 2008

\begin{abstract}
We present almost sure central limit theorems for stochastic processes whose time parameter ranges over the d-dimensional unit cube. Our purpose here is to generalize the classic functional central limit theorem of Prokhorov (1956) for such processes. We prove multidimensional analogues of Glivenko-Cantelli type theorems.
\end{abstract}

Key words: almost sure central limit theorem, multiparameter stochastic process, Glivenko-Cantelli type theorem, logarithmic averages

PACS: $02.50 . \mathrm{Cw}$

\section{Introduction and notations}

There has recently been considerable interest in questions of weak convergence of sequences of stochastic processes $\left\{X_{n}(t), n \geqslant 1\right\}$, where $t$ ranges over the unit cube in d-dimensional space. Situations in which such convergence arises include, for example, weak convergence of the normalized empirical cumulative distribution function for samples from a continuous distribution concentrating on the unit cube $\mathrm{R}^{d}$, weak convergence of the normalized, randomly-stopped empirical cumulative for samples from a d-dimensional continuous distribution on the unit d-cube, convergence of the analogue of partial sum processes for d-dimensional time, cf., Wichura [25], Bickel and Wichura [5], Pyke [19], Kuelbs [14].

On the other hand, starting with Brosamler [7] and Schatte [22], in the past decade several authors have investigated the almost sure central limit theorems and related 'logarithmic' limit theorems for partial sums of independent and dependent random variables. A survey of pointwise central limit theorems can be found in Berkes [3], and Berkes and Csáki [4].

Some functional versions of the almost sure central limit theorem have also been presented, cf. Brosamler [7], Lacey and Philipp [15], Schatte [22-24], Atlagh [1], Rodzik and Rychlik [20], Rychlik and Szuster [21].

The purpose of this paper is to extend the almost sure central limit theorems for sequences of random variables to sequences of stochastic processes $\left\{X_{n}(t), n \geqslant 1\right\}$, where $t$ ranges over the unit cube in d-dimensional space. Some results, concerning almost sure central limit theorems for random fields, have been presented by Fazekas and Rychlik [9].

In this paper we prove multidimensional analogues of the Glivenko-Cantelli type theorems. We present almost sure versions of the functional central limit theorem, corresponding to weak limit theorems more general, than Theorem 3 of Wichura [25] and Theorem 5 of Bickel and Wichura [5].

The almost sure versions of the central limit theorems can be viewed as a uniform strong law of large numbers or a Glivenko-Cantelli type result, cf., Csörgő and Horváth [8]. Strong laws of

*Supported by the DFG under the Project 436 POL 113/115/0-1. 
large numbers for multiindex sequences need stronger assumptions than strong laws for ordinary sequences, cf., Gut [12]. On the other hand, weak convergence of probability measures is metrizable. Therefore, the case of multiindex sequences does not require extra conditions. However, in the almost sure central limit theorems we consider weak convergence as well as the almost sure convergence. Therefore, the multiindex case has its own, very interesting meaning. Furthermore, the number of methods to prove the strong law of large numbers for sums of random variables is much less in the case of a multidimensional parameter case. For example, the almost sure invariance principle fails to be effective in this case. Thus, in the proof of the main results, we apply purely probabilistic arguments and do not appeal to ergodic theory, as in Brosmaler [7], or to strong invariance principle, as in Schatte [23,24].

Let $Z_{+}^{d}, d \geqslant 1$, be the set of positive integer d-dimensional lattice points. The points in $Z_{+}^{d}$ will be denoted by $\mathbf{m}, \mathbf{n}$, etc., or, sometimes, when necessary, more explicitly by $\left(m_{1}, \ldots, m_{d}\right)$, $\left(n_{1}, \ldots, n_{d}\right)$, etc. Also, for $\mathbf{n}=\left(n_{1}, \ldots, n_{d}\right)$ we define $|\mathbf{n}|=\prod_{i=1}^{d} n_{i}$. We shall write $\mathbf{0}$ and $\mathbf{1}$ for points $(0, \ldots, 0)$ and $(1, \ldots, 1)$, respectively. The set $Z_{+}^{d}$ is partially ordered by stipulating $\mathbf{m} \leqslant \mathbf{n}$ if $m_{i} \leqslant n_{i}$ for each $i, 1 \leqslant i \leqslant d$. Furthermore, we shall write $\mathbf{m}<\mathbf{n}$ if $\mathbf{m} \leqslant \mathbf{n}$ and $m_{i}<n_{i}$ for at least one $i, 1 \leqslant i \leqslant d$. In this paper the limit $\mathbf{n} \rightarrow \infty$ will mean $n_{i} \rightarrow \infty$, for every $i=1, \ldots, d$. On the other hand, the relations $\min$ and $\max$ we define coordinatewise. The limit superior of $\left\{a_{\mathbf{n}}, \mathbf{n} \in Z_{+}^{d}\right\}, \limsup _{\mathbf{n} \rightarrow \infty}$, is to be interpreted as $\inf _{\mathbf{n}} \sup _{\mathbf{n}<\mathbf{m}} a_{\mathbf{m}}$ and similarly for the limit inferior (cf., Gabriel [11], Gut [12,13]).

Let $\left\{X_{\mathbf{n}}, \mathbf{n} \in Z_{+}^{d}\right\}$ be a random field of independent random variables, defined on a probability space

$$
(\Omega, \mathcal{A}, P), \quad \text { such that } \quad E X_{\mathbf{n}}=0, \quad E X_{\mathbf{n}}^{2}=b_{\mathbf{n}}^{2}<\infty, \quad \mathbf{n} \in Z_{+}^{d} .
$$

Let us put

$$
S_{\mathbf{n}}=\sum_{\mathbf{k} \leqslant \mathbf{n}} X_{\mathbf{k}}, \quad B_{\mathbf{n}}^{2}=E S_{\mathbf{n}}^{2}=\sum_{\mathbf{k} \leqslant \mathbf{n}} b_{\mathbf{k}}^{2}, \mathbf{n} \in Z_{+}^{d}
$$

and assume, for every $\epsilon>0$,

$$
B_{\mathbf{n}}^{-2} \sum_{\mathbf{k} \leqslant \mathbf{n}} E X_{\mathbf{k}}^{2} I\left(\left|X_{\mathbf{k}}\right| \geqslant \epsilon B_{\mathbf{n}}\right) \rightarrow 0 \quad \text { as } \quad \mathbf{n} \rightarrow \infty
$$

Relation (1.2) is the exact analogue of the classical Lindeberg's condition and is more general than the one, considered by Bickel and Wichura [5]. On the other hand, if (1.2) holds, then for every $\epsilon>0$

$$
\left(\max _{\mathbf{1} \leqslant \mathbf{k} \leqslant \mathbf{n}} b_{\mathbf{k}}^{2}\right) / B_{\mathbf{n}}^{2} \leqslant \epsilon^{2}+B_{\mathbf{n}}^{-2} \sum_{\mathbf{k} \leqslant \mathbf{n}} E X_{\mathbf{k}}^{2} I\left(\left|X_{\mathbf{k}}\right| \geqslant \epsilon B_{\mathbf{n}}\right) \rightarrow \epsilon^{2} \quad \text { as } \quad \mathbf{n} \rightarrow \infty .
$$

Thus, since $\epsilon>0$ can be chosen arbitrarily small, (1.2) implies

$$
\left(\max _{\mathbf{1} \leqslant \mathbf{k} \leqslant \mathbf{n}} b_{\mathbf{k}}^{2}\right) / B_{\mathbf{n}}^{2} \rightarrow 0 \quad \text { as } \quad \mathbf{n} \rightarrow \infty .
$$

Relation (1.3) is an d-dimensional analogue of the classical Feller's condition.

Let $\left(D[0,1]^{d}, \mathfrak{D}_{d}\right)$ be the Skorkhod space of functions defined on the unit cube $[0,1]^{d}$. With respect to the corresponding metric topology $\left(S-\right.$ topology), $\left(D[0,1]^{d}, \mathfrak{D}_{d}\right)$ is separable and topologically complete, and the Borel $\sigma-$ algebra $\mathfrak{D}_{d}$ coincides with the $\sigma-$ algebra generated by the coordinate mappings, cf., Bickel and Wichura [5], Neuhaus [17], Billingsley [6]. Of course, this metric topology on $D[0,1]^{d}$ for $d=1$ coincides with Skorokhod's well-known and useful $J_{1}-$ topology (see Billingsley [6], for example). The functions in $D[0,1]^{d}$ may be characterized by their continuity properties, as follows. If $\mathbf{t} \in[0,1]^{d}$ and if, for $1 \leqslant p \leqslant d, R_{p}$ is one of the relations $<$ and $\geqslant$, let $Q_{R_{1}, \ldots R_{d}}(\mathbf{t})$ denote the following quadrant

$$
\left\{\left(s_{1}, \ldots, s_{d}\right) \in D[0,1]^{d}: s_{p} R t_{p}, 1 \leqslant p \leqslant d\right\} .
$$


Then (see Neuhaus [17], Bickel and Wichura [5] ) $x \in D[0,1]^{d}$ if and only if, for each $\mathbf{t} \in[\mathbf{0}, \mathbf{1}]^{d}$,

$$
x_{Q} \equiv \lim _{\mathbf{s} \rightarrow \mathbf{t}, \mathbf{s} \in \mathbf{Q}} x(\mathbf{s})
$$

exists for each of the $2^{d}$ quadrants $Q=Q_{R_{1}, \ldots, R_{d}}(\mathbf{t})$, and

$$
x(\mathbf{t})=x_{Q \geqslant, \ldots, \geqslant} .
$$

Thus, in this sense, the functions of $D[0,1]^{d}$ are "continuous from above, with limits from below".

\section{Results}

The following theorem extends the classic result of Lindeberg and Feller.

Theorem 2.1. Let $\left\{X_{\mathbf{n}}, \mathbf{n} \in Z_{+}^{d}\right\}$ be a random field of independent random variables such that $E X_{\mathbf{n}}=0, E X_{\mathbf{n}}^{2}=b_{\mathbf{n}}^{2}<\infty, \mathbf{n} \in Z_{+}^{d}$. Then (1.2) holds if and only if (1.3) holds and

$$
S_{\mathbf{n}} / B_{\mathbf{n}} \Longrightarrow N(0,1) \quad \text { as } \quad \mathbf{n} \rightarrow \infty
$$

where $N(0,1)$ denotes the standard normal distribution.

Here, and subsequently, $\Longrightarrow$ denotes the weak convergence of measures.

We would like to note that some special cases of Theorem 2.1 can be deduced from the results presented by Wichura [25], Bickel and Wichura [5] and Eagodowski and Rychlik [16], but even in those cases only implication (1.2) implies that (2.1) have been proved.

Let $\left\{X_{\mathbf{n}}, \mathbf{n} \in Z_{+}^{d}\right\}$ be a random field of independent random variables with zero means and finite variances. Assume, for each $\mathbf{n}=\left(n_{1}, \ldots, n_{d}\right) \in Z_{+}^{d}$,

$$
E X_{\mathbf{n}}^{2}=b_{n_{1}}^{(1)} b_{n_{2}}^{(2)} \ldots b_{n_{d}}^{(d)}=\prod_{i=1}^{d} b_{n_{i}}^{(i)}
$$

Then, by $(2.2)$, for each $\mathbf{n}=\left(n_{1}, \ldots, n_{d}\right) \in Z_{+}^{d}$ and $\mathbf{k}=\left(k_{1}, \ldots, k_{d}\right) \in Z_{+}^{d}$ we have,

$$
E S_{\mathbf{n}}^{2}=\sum_{\mathbf{k} \leqslant \mathbf{n}} E X_{\mathbf{k}}^{2}=\sum_{\mathbf{k} \leqslant \mathbf{n}} \prod_{i=1}^{d} b_{k_{i}}^{(i)}=\prod_{i=1}^{d} B_{n_{i}}^{(i)}=\left|B_{\mathbf{n}}\right|
$$

where $B_{n_{i}}^{(i)}=\sum_{j=1}^{n_{i}} b_{j}^{(i)}, 1 \leqslant i \leqslant d, B_{\mathbf{n}}=\left(B_{n_{1}}^{(1)}, \ldots, B_{n_{d}}^{(d)}\right)$.

Let, by definition, $X_{\mathbf{0}}=S_{\mathbf{0}}=B_{\mathbf{0}}=\mathbf{0}$. Let $\mathbf{t}=\left(t_{1}, \ldots, t_{d}\right) \in[0,1]^{d}$, and let

$$
m(\mathbf{t})=\max \left\{\mathbf{k} \geqslant \mathbf{0}: B_{\mathbf{k}} \leqslant \mathbf{t}\right\}, \quad M(\mathbf{t})=\min \left\{\mathbf{k} \geqslant \mathbf{0}: \mathbf{t} \leqslant B_{\mathbf{k}}\right\} .
$$

Set

$$
m_{\mathbf{n}}(\mathbf{t})=m\left(\mathbf{t} B_{\mathbf{n}}\right), \quad M_{\mathbf{n}}(\mathbf{t})=M\left(\mathbf{t} B_{\mathbf{n}}\right), \quad \mathbf{t} \in[0,1]^{d},
$$

where, by definition, $\mathbf{t} B_{\mathbf{n}}=\left(t_{1} B_{n_{1}}^{(1)}, \ldots, t_{d} B_{n_{d}}^{(d)}\right)$.

Let, for every $\mathbf{t} \in[0,1]^{d}$ and any $\mathbf{n} \in Z_{+}^{d}$,

$$
Y_{\mathbf{n}}(\mathbf{t})=S_{m_{\mathbf{n}}(\mathbf{t})} /\left|B_{\mathbf{n}}\right|^{1 / 2}
$$

Then $\left\{Y_{\mathbf{n}}, \mathbf{n} \in Z_{+}^{d}\right\}$ is a random field of $D[0,1]^{d}$ - valued random elements.

Let us observe that if (2.2) and (1.2) hold, then Feller's condition (1.3) has the following form

$$
\max _{\mathbf{k} \leqslant \mathbf{n}} \frac{E X_{\mathbf{k}}^{2}}{B_{\mathbf{n}}^{2}}=\max _{\mathbf{k} \leqslant \mathbf{n}} \prod_{i=1}^{d}\left(\frac{b_{k_{i}}^{(i)}}{B_{n_{i}}^{(i)}}\right)=\prod_{i=1}^{d}\left(\max _{1 \leqslant k_{i} \leqslant n_{i}} b_{k_{i}}^{(i)} / B_{n_{i}}^{(i)}\right) \rightarrow 0 \quad \text { as } \quad \mathbf{n} \rightarrow \infty .
$$


In what follows we shall also need the following condition

$$
\max _{1 \leqslant i \leqslant d}\left(\max _{1 \leqslant k_{i} \leqslant n_{i}} b_{k_{i}}^{(i)} / B_{n_{i}}^{(i)}\right) \rightarrow 0 \quad \text { as } \quad \mathbf{n} \rightarrow \infty
$$

Let us observe, that (2.6) is a consequence of (2.7) and also follows from (1.2). On the other hand, in general, (2.7) is not a consequence of (1.2) or (2.6). If (2.7) holds, then we sometimes say that the random field $\left\{Y_{\mathbf{n}}, \mathbf{n} \in Z_{+}^{d}\right\}$ satisfies a stronger version of Feller's condition.

Theorem 2.2. Let $\left\{X_{\mathbf{n}}, \mathbf{n} \in Z_{+}^{d}\right\}$ be a random field of independent random variables with zero means and finite second moments satisfying (2.2). If (1.2) and (2.7) hold, then

$$
Y_{\mathbf{n}} \Longrightarrow W \quad \text { as } \quad \mathbf{n} \rightarrow \infty
$$

where $W=\left\{W(\mathbf{t}): \mathbf{t} \in[0,1]^{d}\right\}$ is a Gaussian process with zero means and covariances

$$
\operatorname{Cov}(W(\mathbf{t}), W(\mathbf{s}))=|\min (\mathbf{t}, \mathbf{s})|=\prod_{i=1}^{d} \min \left(t_{i}, s_{i}\right) .
$$

Let us observe that $W$, in $(2.8)$, is a Brownian motion process on the space $D[0,1]^{d}$. Furthermore, if (2.2) holds, then by $(2.3)$

$$
B_{\mathbf{n}}^{2}=E S_{\mathbf{n}}^{2}=\left|B_{\mathbf{n}}\right|, \quad \mathbf{n} \in Z_{+}^{d} .
$$

Let $\log _{+} x=\log x$, if $x \geqslant e$ and $\log _{+} x=1$, if $x<e$. Let, for $\mathbf{k}=\left(k_{1}, k_{2}, \ldots, k_{d}\right)$ and $\mathbf{n}=\left(n_{1}, n_{2}, \ldots, n_{d}\right)$,

$$
b_{\mathbf{k}}=\prod_{i=1}^{d} b_{k_{i}}^{(i)}, \quad\left|\log _{+} B_{\mathbf{n}}\right|=\prod_{i=1}^{d} \log _{+} B_{n_{i}}^{(i)} .
$$

Let $\zeta_{n}, n \geqslant 1$, be a sequence of random variables defined on a probability space $(\Omega, \mathcal{A}, P)$. Almost sure limit theorems state that

$$
\frac{1}{D_{n}} \sum_{k=1}^{n} d_{k} \delta_{\zeta_{k}(\omega)} \Longrightarrow \mu, \quad \text { as } \quad n \rightarrow \infty, \quad \text { for almost every } \omega \in \Omega
$$

where $\delta_{x}$ is the unit mass at point $x$ and $\Longrightarrow$ denotes weak convergence to the probability measure $\mu$. In the simplest form of the almost sure central limit theorem

$$
\zeta_{k}=\left(X_{1}+\cdots+X_{k}\right) / \sqrt{k}, k \geqslant 1
$$

where $X_{1}, X_{2}, \ldots$, are independent and identically distributed random variables with zero mean and variance one, $d_{k}=1 / k, D_{n}=\log n$, and $\mu$ is the standard normal law $N(0,1)$; see, e.g., Schatte [22,23] and [24]. See also Berkes [3], Atlagh and Weber [2], Berkes and Csáki [4] for surveys.

The general form of the multiindex version of (2.11) is

$$
\frac{1}{D_{\mathbf{n}}} \sum_{\mathbf{k} \leqslant \mathbf{n}} d_{\mathbf{k}} \delta_{\zeta_{\mathbf{k}}} \Longrightarrow \mu, \quad \text { as } \quad \mathbf{n} \rightarrow \infty, \quad \text { for almost every } \quad \omega \in \Omega,
$$

where $\zeta_{\mathbf{k}}, \mathbf{k} \in Z_{+}^{d}$, is a multiindex sequence of random elements.

The multiindex version of the classical central limit theorem is as follows.

Theorem 2.3. Let $\left\{X_{\mathbf{n}}, \mathbf{n} \in Z_{+}^{d}\right\}$ be a random field of independent random variables with zero means and finite second moments satisfying (2.2). If (1.2) and (2.7) hold, then

$$
\frac{1}{\left|\log _{+} B_{\mathbf{n}}\right|} \sum_{\mathbf{k} \leqslant \mathbf{n}} \frac{b_{\mathbf{k}+\mathbf{1}}}{\left|B_{\mathbf{k}}\right|} \delta_{S_{\mathbf{k}}(\omega) /\left|B_{\mathbf{k}}\right|^{1 / 2}} \Longrightarrow N(0,1), \quad \text { as } \quad \mathbf{n} \rightarrow \infty,
$$


for almost every $\omega \in \Omega$.

Let us observe that Theorem 2.3 presents generalization, to sequences of independent nonidentically distributed random variables, Theorem 1.1 obtained by Fazekas and Rychlik [9].

The following Theorem 2.4 is a multiindex version of the classical almost sure functional central limit theorem. In fact it is a multiindex version of the classical almost sure version of Prokhorov's [18] functional central limit theorem. Theorem 2.4 also generalizes Theorem 1.2 presented by Fazekas and Rychlik [9].

Theorem 2.4. Let $\left\{X_{\mathbf{n}}, \mathbf{n} \in Z_{+}^{d}\right\}$ be a random field of independent random variables with zero means and finite second moments satisfying (2.2). If (1.2) and (2.7) hold, then

$$
\frac{1}{\left|\log _{+} B_{\mathbf{n}}\right|} \sum_{\mathbf{k} \leqslant \mathbf{n}} \frac{b_{\mathbf{k}+\mathbf{1}}}{\left|B_{\mathbf{k}}\right|} \delta_{Y_{\mathbf{k}}(\omega)} \Longrightarrow W, \quad \text { as } \quad \mathbf{n} \rightarrow \infty,
$$

for almost every $\omega \in \Omega$, where $W$ is the Wiener measure generated by a Brownian motion process on the space $D[0,1]^{d}$.

Denote the usual integer part by [.], moreover, for $\mathbf{n}=\left(n_{1}, n_{2}, \ldots, n_{d}\right) \in Z_{+}^{d}$ and $\mathbf{t}=\left(t_{1}, t_{2}, \ldots, t_{d}\right) \in[\mathbf{0}, \mathbf{1}]^{d}$ denote the vector $\left(\left[n_{1} t_{1}\right],\left[n_{2} t_{2}\right], \ldots,\left[n_{d} t_{d}\right]\right) \in Z_{+}^{d}$ by $[\mathbf{n t}]$. Then, from Theorems 2.3 and 2.4, we easily get Theorems 1.1 and 1.2 of Fazekas and Rychlik [9].

Theorem 2.5. Let $\left\{X_{\mathbf{n}}, \mathbf{n} \in Z_{+}^{d}\right\}$ be a random field of independent and identically distributed random variables with zero mean and second moment one, then for almost every $\omega \in \Omega$

$$
\frac{1}{\left|\log _{+} \mathbf{n}\right|} \sum_{\mathbf{k} \leqslant \mathbf{n}} \frac{1}{|\mathbf{k}|} \delta_{S_{\mathbf{k}}(\omega) / \sqrt{|\mathbf{k}|}} \Longrightarrow N(0,1), \quad \text { as } \quad \mathbf{n} \rightarrow \infty
$$

and

$$
\frac{1}{\left|\log _{+} \mathbf{n}\right|} \sum_{\mathbf{k} \leqslant \mathbf{n}} \frac{1}{|\mathbf{k}|} \delta_{Y_{\mathbf{k}}^{*}(\omega)} \Longrightarrow W, \quad \text { as } \quad \mathbf{n} \rightarrow \infty
$$

where, for $\mathbf{n}=\left(n_{1}, n_{2}, \ldots, n_{d}\right) \in Z_{+}^{d},\left|\log _{+} \mathbf{n}\right|=\prod_{i=1}^{d} \log _{+} n_{i}$ and $Y_{\mathbf{k}}^{*}(\mathbf{t})=S_{[\mathbf{k t}]} / \sqrt{|\mathbf{k}|}, \mathbf{t} \in[0,1]^{d}, \mathbf{k} \in$ $Z_{+}^{d}$.

\section{Proofs}

\subsection{Proof of Theorem 2.1.}

The proof of Theorem 2.1 is almost the same as the proof of Theorems 1 and 2 presented by Feller [10], p. 518-520, but we present it here for the sake of completeness.

Assume (1.2) holds. Then, as we noted above, (1.3) also holds. Let $\varphi_{\mathbf{k}}$ and $F_{\mathbf{k}}$ denote the characteristic function and the distribution function of the random variable $X_{\mathbf{k}}$, respectively. Choose $\zeta>0$ arbitrarily, but fixed. We have to show that

$$
\prod_{\mathbf{k} \leqslant \mathbf{n}} \varphi_{\mathbf{k}}\left(\zeta / B_{\mathbf{n}}\right) \rightarrow \exp \left(-\zeta^{2} / 2\right) \quad \text { as } \quad \mathbf{n} \rightarrow \infty
$$

On the other hand, for every $\mathbf{k}, \mathbf{n} \in Z_{+}^{d}, \mathbf{k} \leqslant \mathbf{n}$, we have

$$
\varphi_{\mathbf{k}}\left(\frac{\zeta}{B_{\mathbf{n}}}\right)-1+\frac{b_{\mathbf{k}}^{2} \zeta^{2}}{2 B_{\mathbf{n}}^{2}}=\int_{-\infty}^{+\infty}\left(\exp \left(i \zeta x / B_{\mathbf{n}}\right)-1-\frac{i \zeta x}{B_{\mathbf{n}}}+\frac{\zeta^{2} x^{2}}{2 B_{\mathbf{n}}^{2}}\right) F_{\mathbf{k}}\{\mathrm{d} x\} .
$$

Furthermore, by (1.3) and Lemma 1 of Feller (1971), p. 512, for every $\epsilon>0$

$$
\left|\varphi_{\mathbf{k}}\left(\frac{\zeta}{B_{\mathbf{n}}}\right)-1\right| \leqslant \frac{\zeta^{2} b_{\mathbf{k}}^{2}}{2 B_{\mathbf{n}}^{2}} \leqslant \zeta^{2}\left(\max _{\mathbf{k} \leqslant \mathbf{n}} b_{\mathbf{k}}^{2}\right) /\left(2 B_{\mathbf{n}}^{2}\right)<\epsilon \zeta^{2}
$$


for $\mathbf{n}$ sufficiently large. Thus, from the Taylor series expansion and (3.3), we get

$$
\sum_{\mathbf{k} \leqslant \mathbf{n}}\left|\log \varphi_{\mathbf{k}}\left(\frac{\zeta}{B_{\mathbf{n}}}\right)-\left(\varphi_{\mathbf{k}}\left(\frac{\zeta}{B_{\mathbf{n}}}\right)-1\right)\right| \leqslant \sum_{\mathbf{k} \leqslant \mathbf{n}}\left|\varphi_{\mathbf{k}}\left(\frac{\zeta}{B_{\mathbf{n}}}\right)-1\right|^{2}<\epsilon \zeta^{2} \sum_{\mathbf{k} \leqslant \mathbf{n}}\left(b_{\mathbf{k}}^{2} / B_{\mathbf{n}}^{2}\right)=\epsilon \zeta^{2}
$$

Hence, by (3.4), for every fixed $\zeta$ we have

$$
\sum_{\mathbf{k} \leqslant \mathbf{n}} \log \varphi_{\mathbf{k}}\left(\frac{\zeta}{B_{\mathbf{n}}}\right) \sim \sum_{\mathbf{k} \leqslant \mathbf{n}}\left(\varphi_{\mathbf{k}}\left(\frac{\zeta}{B_{\mathbf{n}}}\right)-1\right) \quad \text { as } \quad \mathbf{n} \rightarrow \infty
$$

Thus, it is enough to prove that the right hand side of (3.5) tends to $-\frac{\zeta^{2}}{2}$ as $\mathbf{n} \rightarrow \infty$. For this purpose we estimate the integrand in (3.2) by Lemma 1 of Feller [10], p. 512. It follows that for $|x| \leqslant \epsilon B_{\mathbf{n}}$ the integrand is dominated by

$$
\left|\frac{\zeta x}{B_{\mathbf{n}}}\right|^{3} \leqslant \epsilon|\zeta|^{3} x^{2} / B_{\mathbf{n}}^{2}
$$

On the other hand, for $|x|>\epsilon B_{\mathbf{n}}$ we use the upper bound $(\zeta x)^{2} / B_{\mathbf{n}}^{2}$. Hence, we get

$$
\sum_{\mathbf{k} \leqslant \mathbf{n}}\left|\varphi_{\mathbf{k}}\left(\frac{\zeta}{B_{\mathbf{n}}}\right)-1+\frac{b_{\mathbf{k}}^{2} \zeta^{2}}{2 B_{\mathbf{n}}^{2}}\right| \leqslant \epsilon|\zeta|^{3}+\frac{\zeta^{2}}{B_{\mathbf{n}}^{2}} \sum_{\mathbf{k} \leqslant \mathbf{n}} \int_{|x|>\epsilon B_{\mathbf{n}}} x^{2} F_{\mathbf{k}}\{\mathrm{d} x\} .
$$

Since $\epsilon>0$ can be chosen arbitrarily small and (1.2) holds, the right hand side of (3.5) is therefore asymptotically the same as $-\zeta^{2} / 2$, which gives $(2.1)$.

Assume (2.1) and (1.3) hold. If (1.3) holds, then (3.5) also holds. On the other hand, if (2.1) holds, then the imaginary part of the right hand side in (3.5) goes to zero as $\mathbf{n} \rightarrow \infty$. Thus, for every fixed $\epsilon>0$ and any $\zeta$, as $\mathbf{n} \rightarrow \infty$,

$$
\frac{\zeta^{2}}{2}-\sum_{\mathbf{k} \leqslant \mathbf{n}} \int_{|x| \leqslant \epsilon B_{\mathbf{n}}}\left(1-\cos \frac{\zeta x}{B_{\mathbf{n}}}\right) F_{\mathbf{k}}\{\mathrm{d} x\}=\sum_{\mathbf{k} \leqslant \mathbf{n}} \int_{|x|>\epsilon B_{\mathbf{n}}}\left(1-\cos \frac{\zeta x}{B_{\mathbf{n}}}\right) F_{\mathbf{k}}\{\mathrm{d} x\}+o(1) .
$$

Furthermore, the integrand on the right hand side of (3.7) is $\leqslant 2<2 x^{2} /\left(\epsilon B_{\mathbf{n}}\right)^{2}$, and that on the left hand side, $\leqslant \zeta^{2} x^{2} /\left(2 B_{\mathbf{n}}^{2}\right)$. Hence, taking this into account an dividing $(3.7)$ by $\zeta^{2} / 2$, we get

$$
1-\frac{1}{B_{\mathbf{n}}^{2}} \sum_{\mathbf{k} \leqslant \mathbf{n}} \int_{|x| \leqslant \epsilon B_{\mathbf{n}}} x^{2} F_{\mathbf{k}}\{\mathrm{d} x\} \leqslant\left(\frac{4}{\zeta^{2} \epsilon^{2}}\right) \frac{1}{B_{\mathbf{n}}^{2}} \sum_{\mathbf{k} \leqslant \mathbf{n}} \int_{|x|>\epsilon B_{\mathbf{n}}} x^{2} F_{\mathbf{k}}\{d x\}+o(1) \leqslant \frac{4}{\zeta^{2} \epsilon^{2}}+o(1) .
$$

Now, choosing $\zeta$ sufficiently large we see that the right hand side of (3.8) can be made arbitrarily small, and this is evidently the same as (1.2), which completes the proof of Theorem 2.1.

\subsection{Proof of Theorem 2.2.}

We shall first establish that the finite dimensional distributions of $\left\{Y_{\mathbf{n}}, \mathbf{n} \in Z_{+}^{d}\right\}$ weakly converge to the corresponding finite dimensional distributions of $W=\left\{W(\mathbf{t}): \mathbf{t} \in[0,1]^{d}\right\}$.

Let us observe that by Theorem 2.1 , for every $\mathbf{t} \in[0,1]^{d}$, we have

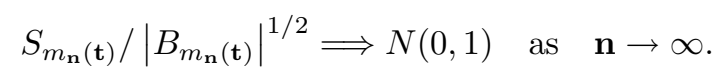

On the other hand, if $\mathbf{t}=\left(t_{1}, \ldots, t_{d}\right)$, then

$$
\left|B_{m_{\mathbf{n}}(\mathbf{t})}\right| /\left|B_{\mathbf{n}}\right| \leqslant|\mathbf{t}|=\prod_{i=1}^{d} t_{i} \leqslant\left|B_{M_{\mathbf{n}}(\mathbf{t})}\right| /\left|B_{\mathbf{n}}\right| \leqslant\left|B_{m_{\mathbf{n}}(\mathbf{t})+\mathbf{1}}\right| /\left|B_{\mathbf{n}}\right|
$$


and, by (2.7), for each $\mathbf{n}=\left(n_{1}, \ldots, n_{d}\right)$,

$$
\begin{aligned}
1 \geqslant\left|B_{\mathbf{n}}\right| /\left|B_{\mathbf{n}+\mathbf{1}}\right| & =\prod\left(B_{n_{i}}^{(i)} / B_{n_{i}+1}^{(i)}\right)=\prod_{i=1}^{d}\left(1-b_{n_{i}+1}^{(i)} / B_{n_{i}+1}^{(i)}\right) \\
& \geqslant \prod_{i=1}^{d}\left(1-\max _{1 \leqslant k \leqslant n_{i}+1} b_{k}^{(i)} / B_{n_{i}+1}^{(i)}\right) \rightarrow 1 \quad \text { as } \quad \mathbf{n} \rightarrow \infty .
\end{aligned}
$$

Thus, by (3.9), (3.10) and (3.11), we get

$$
S_{m_{\mathbf{n}}(\mathbf{t})} /\left|B_{\mathbf{n}}\right|^{1 / 2} \Longrightarrow W(\mathbf{t}) \quad \text { as } \quad \mathbf{n} \rightarrow \infty .
$$

Consider now two time points $\mathbf{s}$ and $\mathbf{t}$ with $\mathbf{0} \leqslant \mathbf{s}<\mathbf{t} \leqslant \mathbf{1}$. It is obvious that the difference

$$
Y_{\mathbf{n}}(\mathbf{t})-Y_{\mathbf{n}}(\mathbf{s})=\left(S_{m_{\mathbf{n}}(\mathbf{t})}-S_{m_{\mathbf{n}}(\mathbf{s})}\right) /\left|B_{\mathbf{n}}\right|^{1 / 2}
$$

is the sum of independent random variables obeying the Lindeberg condition (1.2). Hence, by Theorem 2.1 and $(2.7), Y_{\mathbf{n}}(\mathbf{t})-Y_{\mathbf{n}}(\mathbf{s})$ converges to the normal distribution with the mean zero and variance equal to

$$
\begin{aligned}
\lim _{\mathbf{n} \rightarrow \infty} \prod_{i=1}^{d}\left(t_{i}-s_{i}\right. & \left.-\max _{1 \leqslant i \leqslant d}\left(\max _{1 \leqslant k_{i} \leqslant n_{i}} b_{k_{i}}^{(i)} / B_{n_{i}}^{(i)}\right)\right)=|\mathbf{t}-\mathbf{s}|=\prod_{i=1}^{d}\left(t_{i}-s_{i}\right) \\
& \leqslant \lim _{\mathbf{n} \rightarrow \infty}\left(\left|B_{m_{\mathbf{n}}(\mathbf{t})}\right|-\left|B_{m_{\mathbf{n}}(\mathbf{s})}\right|\right) /\left|B_{\mathbf{n}}\right| \leqslant \lim _{\mathbf{n} \rightarrow \infty} \prod_{i=1}^{d}\left(t_{i}-s_{i}+\left(\max _{1 \leqslant k_{i} \leqslant n_{i}} b_{k_{i}}^{(i)} / B_{n_{i}}^{(i)}\right)\right) \\
& \leqslant \lim _{\mathbf{n} \rightarrow \infty} \prod_{i=1}^{d}\left(t_{i}-s_{i}+\max _{1 \leqslant i \leqslant d}\left(\max _{1 \leqslant k_{i} \leqslant n_{i}} b_{k_{i}}^{(i)} / B_{n_{i}}^{(i)}\right)\right)=|\mathbf{t}-\mathbf{s}|=\prod_{i=1}^{d}\left(t_{i}-s_{i}\right) .
\end{aligned}
$$

Thus, we get

$$
Y_{\mathbf{n}}(\mathbf{t})-Y_{\mathbf{n}}(\mathbf{s}) \Longrightarrow W(\mathbf{t})-W(\mathbf{s}), \quad \text { as } \quad \mathbf{n} \rightarrow \infty .
$$

On the other hand, for all real numbers $c_{1}$ and $c_{2}$

$$
c_{1} Y_{\mathbf{n}}(\mathbf{s})+c_{2}\left(Y_{\mathbf{n}}(\mathbf{t})-Y_{\mathbf{n}}(\mathbf{s})\right)=\left\{c_{1} S_{m_{\mathbf{n}}(\mathbf{s})}+c_{2}\left(S_{m_{\mathbf{n}}(\mathbf{t})}-S_{m_{\mathbf{n}}(\mathbf{s})}\right)\right\} /\left|B_{\mathbf{n}}\right|^{1 / 2},
$$

which is again a sum of independent random variables satisfying the Lindeberg condition (1.2). Thus, by Theorem 2.1, (2.7), (3.13) and 3.14), for all $c_{1}$ and $c_{2}$

$$
c_{1} Y_{\mathbf{n}}(\mathbf{s})+c_{2}\left(Y_{\mathbf{n}}(\mathbf{t})-Y_{\mathbf{n}}(\mathbf{s})\right) \Longrightarrow c_{1} W(\mathbf{s})+c_{2}(W(\mathbf{t})-W(\mathbf{s})) \quad \text { as } \quad \mathbf{n} \rightarrow \infty .
$$

As a consequence of (3.15) and the Cramér-Wald Device, Theorem 7.7 of Billingsley [6], we have

$$
\left(Y_{\mathbf{n}}(\mathbf{s}), Y_{\mathbf{n}}(\mathbf{t})-Y_{\mathbf{n}}(\mathbf{s})\right) \Longrightarrow(W(\mathbf{s}), W(\mathbf{t})-W(\mathbf{s})) \quad \text { as } \quad \mathbf{n} \rightarrow \infty .
$$

Thus, by Corollary 1 to Theorem 5.1 of Billingsley [6], we get

$$
\left(Y_{\mathbf{n}}(\mathbf{s}), Y_{\mathbf{n}}(t)\right) \Longrightarrow(W(\mathbf{s}), W(\mathbf{t})) \quad \text { as } \quad \mathbf{n} \rightarrow \infty
$$

A set of three or more time points can be treated in the same way, and hence the finitedimensional distributions of $\left\{Y_{\mathbf{n}}, \mathbf{n} \in Z_{+}^{d}\right\}$ weakly converge to the corresponding finite-dimensional distributions of $W=\left\{W(\mathbf{t}): \mathbf{t} \in[0,1]^{d}\right\}$.

We shall now show that the random field $\left\{Y_{\mathbf{n}}, \mathbf{n} \in Z_{+}^{d}\right\}$, in $\left(D[0,1]^{d}, \mathfrak{D}_{d}\right)$, is tight. For the tightness of this random field it is enough to prove that there exists a positive number $C$ such that for each $\mathbf{s}$ and $\mathbf{t}$ in $[0,1]^{d}, \mathbf{0} \leqslant \mathbf{s}<\mathbf{t} \leqslant \mathbf{1}$,

$$
\limsup _{\mathbf{n} \rightarrow \infty} E\left|Y_{\mathbf{n}}(\mathbf{t})-Y_{\mathbf{n}}(\mathbf{s})\right|^{2} \leqslant C|\mathbf{t}-\mathbf{s}|=C \prod_{i=1}^{d}\left|t_{i}-s_{i}\right| .
$$


The condition (3.16) is a direct consequence of Theorems 2 and 3 of Wichura [25] or Theorems 2, 3 and 4 of Bickel and Wichura [5].

It is obvious that, by $(1.2),(2.2),(2.3)$ and $(2.7)$, we have

$$
\begin{aligned}
\limsup _{\mathbf{n} \rightarrow \infty} E\left(Y_{\mathbf{n}}(\mathbf{t})-Y_{\mathbf{n}}(\mathbf{s})\right)^{2} & \leqslant \limsup _{\mathbf{n} \rightarrow \infty}\left(\left|B_{m_{\mathbf{n}}(\mathbf{t})}\right|-\left|B_{m_{\mathbf{n}}(\mathbf{s})}\right|\right) /\left|B_{\mathbf{n}}\right| \\
& \leqslant \limsup _{\mathbf{n} \rightarrow \infty} \prod_{i=1}^{d}\left(t_{i}-s_{i}+\left(\max _{1 \leqslant k_{i} \leqslant n_{i}} b_{k_{i}}^{(i)}\right) / B_{n_{i}}^{(i)}\right) \\
& \leqslant \limsup _{\mathbf{n} \rightarrow \infty} \prod_{i=1}^{d}\left(t_{i}-s_{i}+\max _{1 \leqslant i \leqslant d}\left(\max _{1 \leqslant k_{i} \leqslant n_{i}} b_{k_{i}}^{(i)} / B_{n_{i}}^{(i)}\right)\right)=|\mathbf{t}-\mathbf{s}|,
\end{aligned}
$$

which gives (3.16) with $C=1$, and completes the proof of Theorem 2.2.

\subsection{Proof of Theorem 2.3.}

In the proofs of Theorems 2.3 and 2.4 we shall also follow some ideas of Fazekas and Rychlik [9]. Let $(B, \rho)$ be a complete separable metric space and let $\zeta_{\mathbf{n}}, \mathbf{n} \in Z_{+}^{d}$, be a multiindex sequence of random elements in $B$. Let $\mu_{\zeta}$ denote the distribution of $\zeta$.

Lemma 3.1. (Fazekas and Rychlik [9], Theorem 2.1 and Remark 2.2)

Assume that for any pair $\mathbf{h}, \mathbf{l} \in Z_{+}^{d}, \mathbf{h} \leqslant \mathbf{l}$, there exists a $B$ - valued random element $\zeta_{\mathbf{h}, \mathbf{l}}$ with the following properties. If $\mathbf{h}=\mathbf{l}$, then $\zeta_{\mathbf{h}, \mathbf{l}}=0$, and if $\mathbf{k}, \mathbf{l} \in Z_{+}^{d}$, then for $\mathbf{h}=\min \{\mathbf{k}, \mathbf{l}\}$ the following pairs of random elements are independent: $\zeta_{\mathbf{k}}$ and $\zeta_{\mathbf{h}, \mathbf{l}} ; \zeta_{\mathbf{l}}$ and $\zeta_{\mathbf{h}, \mathbf{k}} ; \zeta_{\mathbf{h}, \mathbf{k}}$ and $\zeta_{\mathbf{h}, \mathbf{l}}$. Assume that there exist $C>0, \epsilon>0$, and $d$ increasing sequences $\left\{c_{n}^{(i)}, n \geqslant 1\right\}$, of positive numbers with $\lim _{n \rightarrow \infty} c_{n}^{(i)}=\infty, c_{n+1}^{(i)} / c_{n}^{(i)}=O(1)$, as $n \rightarrow \infty$, for each $i=1,2, \ldots, d$, such that

$$
E\left\{\rho^{2}\left(\zeta_{\mathbf{l}}, \zeta_{\mathbf{h}, 1}\right) \wedge 1\right\} \leqslant C \prod_{i=1}^{d}\left\{\log _{+} \log _{+}\left(\frac{c_{l_{i}}^{(i)}}{c_{h_{i}}^{(i)}}\right)\right\}^{-2(1+\epsilon)}
$$

for $\mathbf{h}=\left(h_{1}, h_{2}, \ldots, h_{d}\right) \leqslant\left(l_{1}, l_{2}, \ldots, l_{d}\right)=1$. Let $0 \leqslant d_{k}^{(i)} \leqslant \log \left(c_{k+1}^{(i)} / c_{k}^{(i)}\right)$, assume that

$$
\sum_{k=1}^{\infty} d_{k}^{(i)}=\infty \quad \text { for } \quad i=1,2, \ldots, d
$$

Let

$$
d_{\mathbf{k}}=\prod_{i=1}^{d} d_{k_{i}}^{(i)} \quad \text { and } \quad D_{\mathbf{n}}=\sum_{\mathbf{k} \leqslant \mathbf{n}} d_{\mathbf{k}}
$$

Then for any probability distribution $\mu$ on the Borel $\sigma$ - algebra of $B$ the following two statements are equivalent

$$
\begin{aligned}
\frac{1}{D_{\mathbf{n}}} \sum_{\mathbf{k} \leqslant \mathbf{n}} d_{\mathbf{k}} \delta_{\zeta_{\mathbf{k}}(\omega)} & \Longrightarrow \mu, \quad \text { as } \quad \mathbf{n} \rightarrow \infty, \quad \text { for almost every } \quad \omega \in \Omega ; \\
\frac{1}{D_{\mathbf{n}}} \sum_{\mathbf{k} \leqslant \mathbf{n}} d_{\mathbf{k}} \mu_{\zeta_{\mathbf{k}}} & \Longrightarrow \mu, \quad \text { as } \quad \mathbf{n} \rightarrow \infty
\end{aligned}
$$

Lemma 3.1 remains valid if condition (3.17) is replaced by the following

$$
E\left\{\rho^{2}\left(\zeta_{\mathbf{l}}, \zeta_{\mathbf{h}, \mathbf{l}}\right) \wedge 1\right\} \leqslant C \prod_{i=1}^{d}\left(\frac{c_{h_{i}}^{(i)}}{c_{l_{i}}^{(i)}}\right)^{\beta}
$$


for $\mathbf{h} \leqslant \mathbf{l}$, where $\beta>0$.

Proof of Theorem 2.3. Let

$$
\zeta_{\mathbf{l}}=\frac{1}{\left|B_{\mathbf{l}}\right|^{1 / 2}} \sum_{\mathbf{k} \leqslant \mathbf{1}} X_{\mathbf{k},} \quad \zeta_{\mathbf{h}, \mathbf{l}}=\frac{1}{\left|B_{\mathbf{l}}\right|^{1 / 2}} \sum_{\mathbf{h}<\mathbf{k} \leqslant \mathbf{1}} X_{\mathbf{k}}
$$

for $\mathbf{h} \leqslant \mathbf{l}$. Thus, the independence conditions are satisfied. Furthermore, by Theorem 2.1,

$$
\zeta_{\mathbf{k}}=\frac{1}{\left|B_{\mathbf{k}}\right|^{1 / 2}} \sum_{\mathbf{l} \leqslant \mathbf{k}} X_{\mathbf{l}} \Longrightarrow N(0,1), \quad \text { as } \quad \mathbf{k} \rightarrow \infty .
$$

On the other hand, we have

$$
E\left\{\zeta_{\mathbf{l}}-\zeta_{\mathbf{h}, \mathbf{l}}\right\}^{2}=\frac{1}{\left|B_{\mathbf{l}}\right|} E\left\{\sum_{\mathbf{k} \leqslant \mathbf{h}} X_{\mathbf{k}}\right\}^{2}=\frac{\left|B_{\mathbf{h}}\right|}{\left|B_{\mathbf{l}}\right|}
$$

Therefore (3.21) holds with $c_{k}^{(i)}=B_{k}^{(i)}, 1 \leqslant i \leqslant d$. Moreover, taking into account (1.2) and (2.7), we have

$$
d_{k}^{(i)}=\log \left(B_{k+1}^{(i)} / B_{k}^{(i)}\right)=\log \left(1+b_{k+1}^{(i)} / B_{k}^{(i)}\right) \backsim b_{k+1}^{(i)} / B_{k}^{(i)} \quad \text { as } \quad k \rightarrow \infty
$$

for every $i=1,2, \ldots, d$. Thus, we can choose $d_{k}^{(i)}=b_{k+1}^{(i)} / B_{k}^{(i)}$ and, in consequence, $d_{\mathbf{k}}=$ $b_{\mathbf{k}+\mathbf{1}} /\left|B_{\mathbf{k}}\right|$. Since

$$
D_{n}^{(i)}=\sum_{k=1}^{n} d_{k}^{(i)}=\sum_{k=1}^{n} \log _{+}\left(B_{k+1}^{(i)} / B_{k}^{(i)}\right)=\log _{+} B_{n+1}^{(i)}-\log b_{1}^{(i)} \backsim \log B_{n}^{(i)}, \quad \text { as } \quad n \rightarrow \infty,
$$

and

$$
D_{\mathbf{n}}=\prod_{i=1}^{d} D_{n_{i}}^{(i)} \backsim \prod_{i=1}^{d} \log B_{n_{i}}^{(i)}, \quad \text { as } \quad \mathbf{n} \rightarrow \infty
$$

therefore

$$
D_{\mathbf{n}}=\prod_{i=1}^{d} \log _{+} B_{n_{i}}^{(i)}=\left|\log _{+} B_{\mathbf{n}}\right|
$$

is an appropriate choice. Thus, Theorem 2.3 is a consequence of (3.21) and Lemma 3.1.

\subsection{Proof of Theorem 2.4}

Let

$$
\zeta_{\mathbf{k}}(\mathbf{t})=Y_{\mathbf{k}}(\mathbf{t})=S_{m_{\mathbf{k}}(\mathbf{t})} /\left|B_{\mathbf{k}}\right|^{1 / 2}, \quad \zeta_{\mathbf{h}, \mathbf{k}}(\mathbf{t})=\frac{1}{\left|B_{\mathbf{k}}\right|^{1 / 2}}\left\{S_{m_{\mathbf{k}}(\mathbf{t})}-S_{\min \left\{\mathbf{h}, m_{\mathbf{k}}(\mathbf{t})\right\}}\right\},
$$

for $\mathbf{h} \leqslant \mathbf{k}, \mathbf{t} \in[0,1]^{d}$. Thus, the independence conditions presented in Lemma 3.1 are satisfied. Furthermore, by Theorem 2.2, we have

$$
Y_{\mathbf{k}} \Longrightarrow W, \quad \text { as } \quad \mathbf{k} \rightarrow \infty
$$

in the space $\left(D[0,1]^{d}, \mathfrak{D}_{d}\right)$. Moreover, by the maximal inequality of Wichura [25], Theorem 1 , we have

$$
E\left\{\rho^{2}\left(\zeta_{\mathbf{k}}, \zeta_{\mathbf{h}, \mathbf{k}}\right)\right\} \leqslant \frac{1}{\left|B_{\mathbf{k}}\right|} E\left\{\sup _{\mathbf{j} \leqslant \mathbf{h}} S_{\mathbf{j}}^{2}\right\} \leqslant \frac{4^{d} E S_{\mathbf{h}}^{2}}{\left|B_{\mathbf{k}}\right|}=4^{d} \frac{\left|B_{\mathbf{h}}\right|}{\left|B_{\mathbf{k}}\right|} .
$$

Therefore (3.21) is satisfied with $c_{k}^{(i)}=B_{k}^{(i)}, 1 \leqslant i \leqslant d$. Thus, similarly to the proof of Theorem 2.3, we easily note that Theorem 2.4 is a consequence of (1.2), (2.7), Lemma 3.1 and inequality (3.21) and the proof is complete. 


\subsection{Proof of Theorem 2.5}

Under the conditions of Theorem 3.5, in $(2.2)$ we can take $b_{n_{i}}^{(i)}=1, n_{i} \geqslant 1,1 \leqslant i \leqslant d$.

Thus, if $\mathbf{n} \in Z_{+}^{d}, \mathbf{t} \in[0,1]^{d}$, then $m_{\mathbf{n}}(\mathbf{t})=[\mathbf{n t}]$ and $\left|B_{\mathbf{n}}\right|=|\mathbf{n}|$. Therefore Theorem 2.5 is a consequence of Theorems 2.3 and 2.4 .

\section{References}

1. Atlagh M. Théorème central limite presque sûr et loi du logarithme itéré pour des sommes de variables aléatoires indépendantes, C. R. Acad. Sci. Paris, Sér. I, Probability Theory, 1993, 316, 929-933.

2. Atlagh M., Weber M. Le théorème central limite presque sûr, Expo. Math., 2000, 18, 97-126.

3. Berkes I., Results and problems related to the poinwise central limit theorem, Asymptotic Methods in Probability and Statistics. In: Szyszkowicz, B. (Ed.), A Volume in Honour of Miklós Csörgő. Elsevier, Amsterdam, 1998, 59-96.

4. Berkes I., Csáki E. A universal result in almost sure central limit theory, Stoch. Proc. Appl., 2001, 94, 105-134.

5. Bickel P.J., Wichura M.J., Convergence criteria for multiparameter stochastic processes and some applications, Ann. Math. Statist., 1971, 42, 1656-1670.

6. Billingsley P. Convergence of Probability Measures. Wiley, New York, 1968.

7. Brosamler G.A., An almost everywhere central limit theorem, Math. Proc. Cambridge Philos. Soc., 1988, 104, 561-574.

8. Csörgő M., Horváth L. Invariance principles for logarithmic averages, Math. Proc. Cambridge Philos. Soc., 1992, 112, 195-205.

9. Fazekas I., Rychlik Z. Almost sure central limit theorems for random fields, Math. Nachr., 2003, 259, $12-18$.

10. Feller W. An introduction to probability theory and its applications, second ed., vol. 2. Wiley, New York, 1971.

11. Gabriel J.P., Martingales with a countable filtering index set, Ann. Probab., 1977, 5, 888-898.

12. Gut A. Strong laws for independent identically distributed random variables indexed by a sector, Ann. Probab., 1983, 11, 369-577.

13. Gut A., Marcinkiewicz laws and convergence rates in the law of large numbers for random variables with multidimensional indices, Ann. Probab., 1978, 6, 469-482.

14. Kuelbs J., The invariance principle for a lattice of random variables, Ann. Math. Statist., 1968, 39, $382-389$.

15. Lacey M.T., Philipp W., A note on the almost sure central limit theorem, Statist. Probab. Lett., 1990, 9, 201-214.

16. Łagodowski Z.A., Rychlik Z., Weak convergence of sequences of random elements with multidimensional random indices, Publicationes Mathematicae, 1990, 37, 35-40.

17. Neuhaus G., On weak convergence of stochastic processes with multidimensional time parameter, Ann. Math. Statist., 1971, 42, 1285-1295.

18. Yu.V. Prokhorov, Convergence of random processes and limit theorems in probability theory, Teor. Veroyatnost. i Primenen., 1956, 1, 177-238; English translation in: Theory Probab. Appl., 1956, 1, 157-214.

19. Pyke R., The weak convergence of the empirical process with random sample size, Proc. Cambridge Philos. Soc., 1968, 64, 155-160.

20. Rodzik B., Rychlik Z., An almost sure central limit theorem for independent random variables, Ann. Inst. H. Poincaré, 1994, 30, 1-11..

21. Rychlik Z., Szuster K., Some remarks on the almost sure central limit theorem for independent random variables, Probab. Math. Statist., 2003, 23, 241-249.

22. Schatte P., On strong versions of the central limit theorem, Math. Nachr., 1988, 137, 249-256.

23. Schatte P., On the central limit theorem with almost sure convergence, Probab. Math. Statist., 1991, 11, 315-343.

24. Schatte P., Two remarks on the almost sure central limit theorem, Math. Nachr., 1991, 154, 225-229.

25. Wichura M.J., Inequalities with applications to the weak convergence of random processes with multidimensional time parameters, Ann. Math. Statist., 1969, 40, 681-687. 
Майже певні функціональні центральні граничні теореми для багатопараметричних стохастичних процесів

\author{
Є.Б.Черебак-Мрозович ${ }^{1}$, З.Рихлік², М.Урбанек² \\ 1 Факультет математики, Технічний університет Жешува, Польща \\ 2 Інститут математики, Університет Марії Кюрі-Склодовської, Люблін, Польща \\ Отримано 31 січня 2008 р.
}

Ми подаємо майже певні центральні граничні теореми для стохастичних процесів з часовим параметром, що змінюється у $\alpha$-вимірному одиничному кубі. Нашою метою $є$ узагальнення класичної функціональної центральної граничної теореми Прохорова (1956) для таких процесів. Ми доводимо багатовимірні аналоги теореми типу Глівенко-Кантелі.

Ключові слова: майже певна центральна гранична теорема, багатопараметричний стохастичний процес, теорема типу Глівенко-Кантелі, логарифмічне середнє

PACS: $02.50 . \mathrm{Cw}$ 
